\title{
Article \\ Microglia Influence Neurofilament Deposition in ALS iPSC-Derived Motor Neurons
}

\author{
Reilly L. Allison ${ }^{1}{ }^{(D)}$, Jacob W. Adelman ${ }^{1}$ D, Jenica Abrudan ${ }^{2}$, Raul A. Urrutia ${ }^{2,3,4}$, Michael T. Zimmermann ${ }^{2,4,5}$, \\ Angela J. Mathison 2,3 ${ }^{\mathbb{D}}$ and Allison D. Ebert ${ }^{1, *}$
}

1 Department of Cell Biology, Neurobiology and Anatomy, Medical College of Wisconsin, Milwaukee, WI 53226, USA; rallison@mcw.edu (R.L.A.); jadelman@mcw.edu (J.W.A.)

2 Genomic Science and Precision Medicine Center (GSPMC), Medical College of Wisconsin, Milwaukee, WI 53226, USA; pabrudan@mcw.edu (J.A.); rurrutia@mcw.edu (R.A.U.); mtzimmermann@mcw.edu (M.T.Z.); amathison@mcw.edu (A.J.M.)

3 Division of Research, Department of Surgery, Medical College of Wisconsin, Milwaukee, WI 53226, USA

4 Department of Biochemistry, Medical College of Wisconsin, Milwaukee, WI 53226, USA

5 Clinical and Translational Sciences Institute, Medical College of Wisconsin, Milwaukee, WI 53226, USA

* Correspondence: aebert@mcw.edu; Tel.: +414-955-2979

check for updates

Citation: Allison, R.L.; Adelman, J.W.; Abrudan, J.; Urrutia, R.A.; Zimmermann, M.T.; Mathison, A.J.; Ebert, A.D. Microglia Influence Neurofilament Deposition in ALS iPSC-Derived Motor Neurons. Genes 2022, 13, 241. https://doi.org/ 10.3390 /genes13020241

Academic Editor: Christopher Grunseich

Received: 30 December 2021

Accepted: 25 January 2022

Published: 27 January 2022

Publisher's Note: MDPI stays neutral with regard to jurisdictional claims in published maps and institutional affiliations.

Copyright: () 2022 by the authors. Licensee MDPI, Basel, Switzerland. This article is an open access article distributed under the terms and conditions of the Creative Commons Attribution (CC BY) license (https:/ / creativecommons.org/licenses/by/ $4.0 /)$.

\begin{abstract}
Amyotrophic lateral sclerosis (ALS) is a fatal neurodegenerative disease in which upper and lower motor neuron loss is the primary phenotype, leading to muscle weakness and wasting, respiratory failure, and death. Although a portion of ALS cases are linked to one of over 50 unique genes, the vast majority of cases are sporadic in nature. However, the mechanisms underlying the motor neuron loss in either familial or sporadic ALS are not entirely clear. Here, we used induced pluripotent stem cells derived from a set of identical twin brothers discordant for ALS to assess the role of astrocytes and microglia on the expression and accumulation of neurofilament proteins in motor neurons. We found that motor neurons derived from the affected twin which exhibited increased transcript levels of all three neurofilament isoforms and increased expression of phosphorylated neurofilament puncta. We further found that treatment of the motor neurons with astrocyte-conditioned medium and microglial-conditioned medium significantly impacted neurofilament deposition. Together, these data suggest that glial-secreted factors can alter neurofilament pathology in ALS iPSC-derived motor neurons.
\end{abstract}

Keywords: induced pluripotent stem cells; amyotrophic lateral sclerosis; astrocytes; RNA sequencing; cytokines; anti-inflammatory; glia

\section{Introduction}

Amyotrophic lateral sclerosis (ALS) is a severe and always fatal neurodegenerative disease in which upper and lower motor neurons are lost leading to muscle weakness, paralysis, and death typically within 2-5 years of diagnosis. ALS has been causally linked to several different genetic mutations, but the vast majority of cases are still sporadic in nature with no known genetic cause [1]. Although various mechanisms have been postulated as leading to the motor neuron loss [1], there is still a lack of clear understanding of motor neuron degeneration in ALS.

Despite an unknown cause of motor neuron loss, intracellular inclusions of aggregated proteins are clear pathological hallmarks that span both sporadic and familial ALS patient samples [2]. Aggregates containing TDP-43 are found in greater than $90 \%$ of patients [3], but SOD1, optineurin (OPTN), ubiquitin, and neurofilament are also commonly found $[2,4,5]$. Protein aggregation may contribute to motor neuron death through activation of oxidative stress and/or by causing general cellular dysfunction through sequestering cytosolic proteins to the aggregates themselves [6-9]. The alteration of neurofilament level is of particular interest in ALS because neurofilament is found in high abundance in the cerebral 
spinal fluid from ALS patients and may serve as a key biomarker to ALS disease onset, progression, and therapeutic success [10-12].

Neurofilaments (NFs) are intermediate filament proteins consisting of heteropolymers of the heavy $(\mathrm{H})$, medium $(\mathrm{M})$, and light $(\mathrm{L})$ subunits $[13,14]$. NFs are abundantly expressed in neurons, and they provide structural support, facilitate organelle transport, participate in intracellular signaling, and regulate axon growth $[15,16]$. Mutations in NEFH have been found in a small number of ALS cases [5], but the role of NF proteins in ALS have more recently focused on the potentially pathogenic nature of NF neuronal accumulations [11]. For example, high expression of human NF-H proteins in mice caused large inclusions in motor neurons and contributed to axonal atrophy, diminished conductance, and severe motor deficits [17,18]. Additionally, NF aggregates have been observed in human stem cell models of ALS from both sporadic and familial forms of the disease [19-21]. The mechanisms underlying NF aggregation in ALS are still not fully elucidated, but evidence suggests that excitotoxicity increases NF hyperphosphorylation in neurons [22], thereby promoting NF aggregation.

Increased neuronal excitotoxicity has long been a proposed mechanism associated with motor neuron loss in ALS [23,24]. Astrocytes play a key role in reducing excess glutamate in the synaptic cleft that can lead to neuronal excitotoxicity, but evidence indicates that ALS astrocytes are dysfunctional and actively induce motor neuron toxicity [23,24]. For example, the presence of ALS astrocytes and/or their secreted proteins are sufficient to induce motor neuron damage and loss, both in vitro and in vivo through non-cell autonomous mechanisms [25-30]. Similarly, ALS microglia have been shown to exhibit altered morphology and functionality in vitro and in vivo [31-34]. Interestingly, malfunctioning microglia are also able to induce astrocyte toxicity [35], suggesting a tight interplay between glial cells in health and disease.

Considering the mostly sporadic nature of ALS, having appropriate model systems can be a challenge. While induced pluripotent stem cells (iPSCs) derived from sporadic ALS patients offer a unique opportunity to assess disease properties in specific patient backgrounds, they also prevent the use of isogenic controls as there are no specific gene mutations to correct. To circumvent this problem, we previously generated and characterized iPSCs from identical twin brothers who are discordant for ALS [36]. In our previous studies, we did not find disease-relevant genetic variations between the brothers [36], suggesting that mechanisms at the cellular level may be more influential to disease processes. Therefore, here we used RNA sequencing on the iPSC-derived motor neurons to assess whether there were specific molecular pathways altered in the affected twin that could contribute to the affected brother's ALS diagnosis. Although there were several transcriptional differences between the brothers, we focused our attention on neurofilament due to its relationship to ALS disease pathology. Interestingly, we found that the affected twin exhibited an increased transcript expression of all three neurofilament subtypes as well as neurofilament aggregation in the iPSC-derived motor neurons. Moreover, we found that treating the affected motor neurons with microglia derived from the healthy brother's iPSCs significantly reduced the neurofilament deposition. Together, these data suggest that glial-motor neuron interactions are important modulators of neurofilament pathology in ALS.

\section{Materials and Methods}

\subsection{Pluripotent Stem Cells}

iPSC lines were previously generated from identical twins discordant for ALS (HB19.2, AB34.12) under an approved IRB protocol (PRO00024167). Details about the individuals, iPSC generation, and pluripotency characterization are described in our previous work [36]. iPSCs were maintained on Matrigel (Corning, Corning, NY, USA) in Essential 8 (Gibco, Waltham, MA, USA) and passaged every $4-6$ days. The iPSCs and differentiated cells were confirmed mycoplasma negative. 


\subsection{Motor Neuron Differentiation and Treatments}

Spinal motor neurons were differentiated using a previously published protocol [37]. Differentiation reagents were purchased from ThermoFisher (Waltham, MA, USA) unless noted otherwise. Briefly, embryoid bodies were generated from iPSCs and patterned in the presence of Chir-99021 with dual SMAD inhibition (SB 431542 and LDN 1931899), followed by treatment with retinoic acid (RA), smoothened agonist (SAG), and DAPT in differentiation medium (50\% DMEM/F12, 50\% Neurobasal, 2\% B27, 1\% N2, 1\% Antibiotic/Antimycotic, 0.1\% B-mercaptoethanol, $50 \mathrm{ng} / \mathrm{mL}$ Laminin, $0.5 \mu \mathrm{M}$ Ascorbic Acid, and $10 \mathrm{mM}$ Y-27632). Spinal motor neuron progenitor cells were then dissociated with TrypLE and plated on Matrigel-coated glass coverslips (ICC) or 6-well plates (protein/RNA collection) for terminal differentiation and maturation in growth-factor-supplemented differentiation medium (listed above) for 28-42 days in vitro. Treatments with ACM were performed with $20 \%$ ACM in growth-factor-supplemented media after day 28 and left for $48 \mathrm{~h}$ at $37^{\circ} \mathrm{C}$ before fixing, collection, or analyses.

\subsection{Glial Differentiation and Treatments}

Spinal cord patterned astrocytes were generated from iPSC-derived neural progenitor cells (NPCs) [38]. Differentiation reagents were purchased from ThermoFisher unless otherwise noted. Briefly, iPSCs were grown to confluency, dissociated with Accutase, and plated at 2 million cells/well into Matrigel (Corning)-coated 6-well plates in NPC base medium (50\% DMEM/F12, 50\% Neurobasal, 2\% B27, 1\% N2, 1\% antibiotic-antimycotic, $0.1 \%$ B-mercaptoethanol, $50 \mathrm{ng} / \mathrm{mL}$ laminin, and $0.5 \mu \mathrm{M}$ ascorbic acid) supplemented with $10 \mu \mathrm{M}$ Y-27632, $3 \mu \mathrm{M}$ Chir-99021, $40 \mu \mathrm{M}$ SB431542, and 0.2 $\mu \mathrm{M}$ LDN193189. On day 1, NPC media were changed and supplemented with $3 \mu \mathrm{M}$ Chir-99021, $40 \mu \mathrm{M} \mathrm{SB} 431542$, and $0.2 \mu \mathrm{M}$ LDN193189. On days 2 and 3, NPC media were supplemented with $3 \mu \mathrm{M}$ Chir-99021, $40 \mu \mathrm{M}$ SB431542, $0.2 \mu \mathrm{M}$ LDN193189, $100 \mathrm{nM}$ retinoic acid (RA), and $500 \mathrm{nM}$ hedgehog smoothened agonist (SAG). From day 4 onwards, NPC media were supplemented with $40 \mu \mathrm{M}$ SB431542, $0.2 \mu \mathrm{M}$ LDN193189, 100 nM RA, and 500 nM SAG, and changed daily. NPCs were passaged via Accutase treatment on day 6 (P1), day 12 (P2), and day 18 (P3). P3 NPCs were used for astrocyte differentiations and cultured in ScienCell Astrocyte Medium (ScienCell Research Laboratories, Carlsbad, CA, USA) containing 1\% astrocyte growth supplement, $1 \%$ penicillin-streptomycin, and 2\% B27. Cells were fed every $48 \mathrm{~h}$ and passaged with Accutase every 6-9 days upon confluency (minimum of 3 passages). Passage 4 cells were considered fully differentiated and seeded onto T75 tissue culture flasks for ACM generation and collection.

Microglia were differentiated using the commercially available differentiation kit (StemCell Technologies \#05310,\#100-0019, \#100-0020, Vancouver, BC, Canada) based on a previously published protocol [39]. Briefly, iPSCs were differentiated into hematopoietic progenitor cells (HPCs) using the STEMdiff Hematopoietic Kit (StemCell Technologies, Vancouver, BC, Canada). Floating HPCs were then collected and plated at 50,000 cells $/ \mathrm{mL}$ in STEMdiff microglia differentiation media (StemCell Technologies, Vancouver, BC, Canada) for 24 days, followed by rapid maturation in STEMdiff microglia maturation media (StemCell Technologies, Vancouver, BC, Canada) for at least 4 days. MCM was collected after day 28 , spun to remove cells or debris, and stored in sterile falcon tubes at $-20^{\circ} \mathrm{C}$. Frozen medias were slowly thawed on ice before use.

Astrocyte-conditioned media were collected upon each media change after P4, spun to remove any cells or debris, and stored in sterile Falcon tubes at $-20{ }^{\circ} \mathrm{C}$. Frozen media were slowly thawed on ice before use. Treatments with MCM were performed with 20\% MCM in supplemented Astrocyte media for $48 \mathrm{~h}$ before removal of treatment media. Cells were then rinsed with PBS and fed with supplemented Astrocyte media. Media were collected from treated astrocytes after $48 \mathrm{~h}$. 


\subsection{RNA Sequencing}

Cell pellets were harvested at 3 weeks of differentiation from both affected (quadruplicate) and unaffected (duplicate) differentiations, and transcriptome (RNAseq) analysis was completed at MCW's Genomic Science and Precision Medicine Center (GSPMC). Total RNA was isolated according to manufacturer's instructions (Qiagen, RNeasy Mini Kit, Germantown, MD, USA) with on-column DNA digestion and quality assessed by fragment analysis (Agilent, Santa Clara, CA, USA). All samples were good-quality RNA (RIN > 8.3), and $500 \mathrm{ng}$ input was aliquoted for library preparation. Libraries were prepared according to manufacturer's protocols (TruSeq stranded mRNA, Illumina, San Diego, CA, USA) with a final quality check completed by fragment analysis (Agilent) and quantification by qPCR (Kapa Library Quantification Kit, Kapa Biosystems, Wilmington, MA, USA). Samples were multiplexed and sequenced on a NovaSeq6000 SP flow cell, with $2 \times 100$ bp read lengths captured. Sequencing reads were processed through the MAPR-Seq workflow [40] with differential expression analysis completed with Bioconductor, edgeR v 3.8.6 software [41]. Genes with a false discovery rate (FDR) less than $5 \%$ and an absolute fold change $\geq 2$ were considered significantly differentially expressed. Data were deposited in the Gene Expression Omnibus (GEO) database (GEO accession number GSE192755).

\subsection{Western Blot}

Cell pellets were lysed by snap-freezing and vortexing with Triton X-100, and then the protein concentration was determined using a BCA assay (ThermoFisher). Equal amounts of protein were loaded onto $10 \%$ or $12 \%$ pre-cast Tris- $\mathrm{HCl}$ Mini-PROTEAN gels (Bio-Rad, Hercules, CA, USA) and proteins separated by electrophoresis, then transferred to PVDF membranes (Bio-Rad). Membranes were blocked for $1 \mathrm{~h}$ in Odyssey TBS blocking buffer (LI-COR), followed by overnight primary antibody incubation and $30 \mathrm{~min}$ secondary antibody incubation. Quantification was performed with FIJI (ImageJ, National Institutes of Health, Bethesda, MD, USA) and normalized to REVERT total protein stain (LI-COR). Primary antibodies used were rabbit anti-NF200 (Sigma, N4142, used at 1:2000 dilution, St. Louis, MO, USA), mouse anti-NF145 (Sigma, MAB1621, used at 1:500 dilution, St. Louis, MO, USA), and mouse anti-NF68 (Sigma, N5139, used at 1:500 dilution, St. Louis, MO, USA). Secondary antibodies used were anti-rabbit IRDye 800CW (LI-COR, 1:5000 dilution, Lincoln, NE, USA) and anti-mouse IRDye 680RD (LI-COR, 1:5000 dilution, Lincoln, NE, USA).

Soluble and insoluble fractionation (used for Supplementary Figure S2) was performed as previously described [42]. Briefly, samples were lysed using sonication with Triton-X before centrifugation at $14,000 \mathrm{rpm}$ for $1 \mathrm{~h}$ at $4{ }^{\circ} \mathrm{C}$. Supernatant was removed (soluble fraction) before the insoluble fraction was washed and pelleted $3 \times$ before resuspension in Triton- $X$ with $2 \%$ SDS. Soluble and insoluble fractions were then analyzed via Western blot as described above.

\subsection{Immunocytochemistry}

Plated cells were fixed in $4 \%$ paraformaldehyde (PFA) for $20 \mathrm{~min}$ at room temperature and rinsed with PBS. Nonspecific labeling was blocked and the cells permeabilized with $0.25 \%$ Triton X-100 in PBS with 1\% BSA and $0.1 \%$ Tween 20 for 15 min at room temperature. Cells were incubated with primary antibodies overnight at $4{ }^{\circ} \mathrm{C}$, then labeled with appropriate fluorescently-tagged secondary antibodies. Hoechst nuclear dye was used to label nuclei. Primary antibodies used were goat anti-choline acetyltransferase (ChAT; Sigma, AB144P, 1:50 dilution, St. Louis, MO, USA), rabbit anti-neurofilament 200 (NF200; Sigma, N4142, 1:500 dilution, St. Louis, MO, USA), and mouse anti-phosphoNF (Sigma, MAB1592, 1:300 dilution, St. Louis, MO, USA). This phosphoNF antibody recognizes NF200 and NF145 at low concentrations, with some reactivity to NF68 at high concentrations. Secondary antibodies used were donkey anti-mouse AF488 (Invitrogen, Waltham, MA, USA), goat anti-chicken AF568 (Invitrogen, Waltham, MA, USA), donkey anti-rabbit AF546 
(Invitrogen, Waltham, MA, USA), and donkey anti-mouse AF647 (Invitrogen, Waltham, MA, USA). All secondaries were used at 1:1000 dilution.

Analyses were performed on three randomly selected fields per coverslip using standard fluorescent microscopy and equivalent exposure conditions at $20 \times$. Images were analyzed for total fluorescence in each channel using FIJI (ImageJ) software. NF200 and total phNF stains were normalized to ChAT in order to account for differences in cell densities between regions of interest. phNF+ aggregates were confirmed to colocalize with ChAT stain and were analyzed using Nikon Elements Object Count function. On the software, thresholds were set to identify aggregates with a $3 \times$ greater intensity than average $\mathrm{phNF}$ stain intensity, and restrictions were set for size and circularity (area $\max =259.51 \mu \mathrm{m}^{2}$, circularity $\mathrm{min}=0.50)$. phNF+ aggregates were represented as a percentage of normalized phNF stains for each image to account for differences in cell densities between regions of interest. Representative images were acquired using a confocal microscope with a $63 \times$ oil objective and were displayed as the maximum intensity projection (MIP) of a z-stack of images.

\subsection{Multiplex Cytokine Array}

Eve Technologies (Calgary, AB, Canada) performed the 48 multiplex cytokine array assay from duplicate differentiations using conditioned medium samples generated from iPSC-derived astrocytes and microglia.

\subsection{Statistical Analyses}

$\mathrm{WB}$ and ICC experiments were performed in triplicate on a minimum of two biological replicates. The multiplex cytokine array was performed on samples from two biological replicates for each condition. Data were analyzed using GraphPad Prism software and the appropriate statistical tests including the Student's $t$-test and 1-way ANOVA followed by Tukey's post-hoc analysis of significance. Changes were considered statistically significant when $p<0.05$.

\section{Results}

In order to model sporadic ALS, we previously generated iPSCs from identical twin brothers discordant for ALS [36], which closely resembles an isogenic iPSC pair. Our previous study used whole genome sequencing and found no substantial genetic differences or mutations in known ALS-associated genes between the brothers that would specifically indicate a genetic basis for the affected brother's ALS diagnosis [36]. Moreover, we found no differences in motor neuron survival or response to glutamate stress when comparing iPSCderived motor neurons between the affected and unaffected twins [36]. Therefore, here we used RNA sequencing on the motor neurons to get a better global picture of transcriptional signatures between the affected and unaffected cells to identify molecular pathways that may be involved in motor neuron malfunction and loss. We found 40,418 transcripts to be differentially expressed between the cells, with 3086 significantly upregulated and 2321 significantly downregulated in the affected motor neurons compared to the healthy control (Figure 1A,B). Gene ontology analysis revealed alterations in a variety of molecular function gene groups in the affected twin, including cytoskeletal protein binding and gated channel activity $(-\log (p$-value $)=20.2$ and 17.2, respectively $)$. Likewise, cellular component gene groups - such as those pertaining to the synapse $((-\log (p$-value $)=63.7)$ and cell junctions $((-\log (p$-value $)=58.2)$ - were also found to be affected (Figure 1C). The top five molecular function and cellular component gene groups altered in the affected twin compared to the unaffected twin are shown (Figure 1C). Further ingenuity pathway analysis (IPA) identified CREB signaling in neurons and SNARE signaling as the two most altered pathways with a $-\log (p$-value) of 12.2 and 10.5, respectively (Figure 1D). ALS signaling, with a $-\log (p$-value) of 7.8 , was also highly altered based on pathway analysis (Figure 1D). When assessing the genes associated with the ALS signaling pathway, we found that NF$\mathrm{H}$ (NEFH), NF-M (NEFM), and NF-L (NEFL) were all significantly upregulated in the 
affected brother $(\mathrm{AB})$ motor neurons compared to the healthy brother $(\mathrm{HB})$ (Figure 1A,B). Furthermore, NF-H had a log2 fold change of 1.58 ( $p$-adj $=0.0006$ ), NF-M had a $\log 2$ fold change of 1.9 ( $p$-adj $=0.0031)$, and NF-L had a log2 fold change of 1.81 ( $p$-adj $=0.0006)$. In contrast, peripherin (PRPH), a neuronal intermediate filament protein found in neurons of the peripheral nervous system and in central nervous system neurons with projections to the periphery, was unchanged between the affected and unaffected motor neurons (Figure 1A). IPA placed these altered NF transcripts in the ALS signaling pathway, showing that upregulation of NFs can lead to neuronal protein inclusions, excitotoxicity, and motor neuron death (Figure 1E).

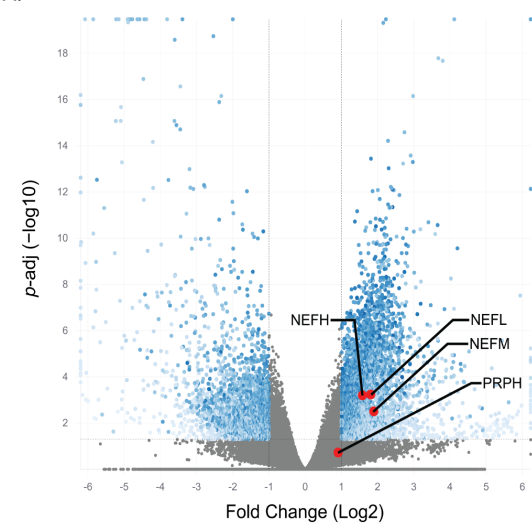

c.
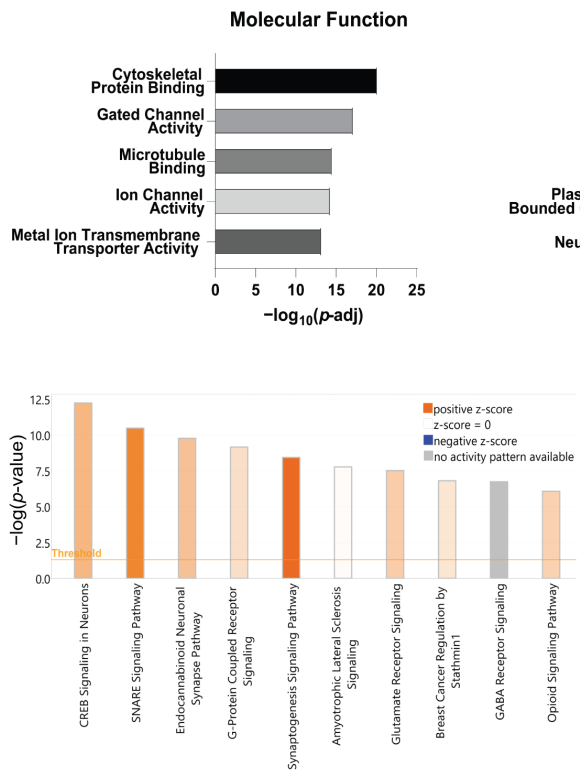

B.

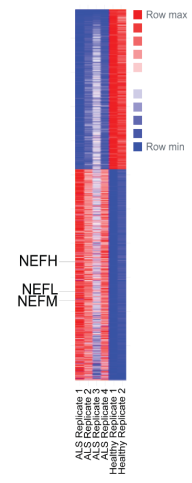

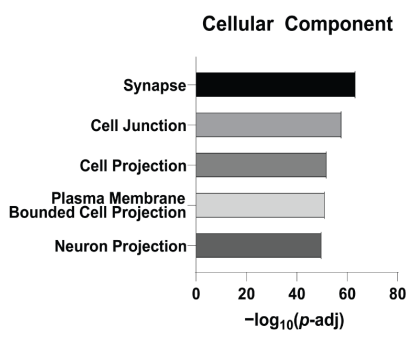

E.

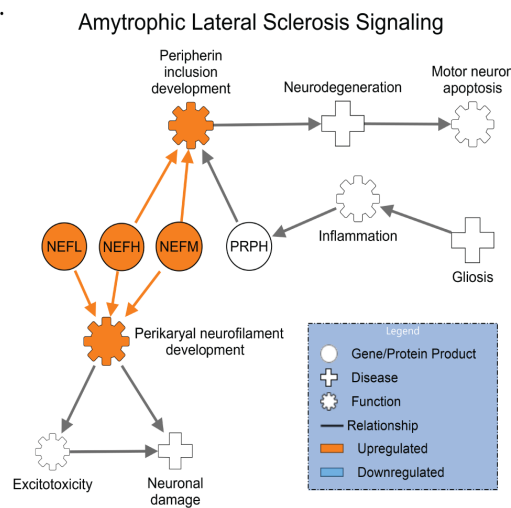

Figure 1. Comparing neurofilament transcripts and ALS-associated signaling in ALS-discordant twins. (A) Volcano plot and (B) heatmap of differential expression RNA sequencing (DESeq2) comparing the motor neuron transcriptome of an ALS-affected patient (AB) to his non-affected twin (HB). Neurofilament transcripts for neurofilament heavy (NEFH), neurofilament medium (NEFM), and neurofilament light (NEFL) are denoted with red dots and labeled [ $p$-adj $<0.0501 \mathrm{FC} \geq \pm 1]$. For comparison, peripherin (PRPH) was unchanged between the AB and HB. (C) Gene ontology identified the top five molecular functions and cellular components altered in $\mathrm{AB}$ motor neurons compared to HB motor neurons [ $p$-adj $<0.0501 \mathrm{FC} \geq \pm 1$ ]. (D) Ingenuity pathway analysis (IPA) highlighting the ten most affected canonical pathways and (E) the role of altered neurofilament gene transcripts in ALS signaling $[p<0.01 ; \mathrm{FC}> \pm 1.35]$. 
With the observed increase in NF noted at the mRNA level, we then assessed protein levels of NF-H (NF200), NF-M (NF145), and NF-L (NF68) using both Western blot and immunocytochemistry. Because the large accumulations of proteins seen at different band sizes in all AB samples (notably around $68 \mathrm{kDa}$ ) could introduce nonspecific antibody binding at these sites, we normalized the samples to REVERT total protein stain rather than a housekeeping gene for more accurate quantification. After this normalization, Western blot analysis did not reveal a difference in NF protein levels in motor neurons between the $\mathrm{AB}$ and the HB for any of the NF subunits (Figure 2A-D). However, using immunocytochemistry, we did observe large accumulations of NF200 staining along the neurites in the $\mathrm{AB}$ motor neuron cultures that were generally absent in the HB motor neurons (Figure 2E,F, arrowheads); these data are similar to what has been observed in mutant SOD1 iPSC-derived motor neurons [20]. However, there was no difference in total NF200 (NF-H) expression in motor neurons between the AB and HB (Figure 2G). We then used immunocytochemistry for phosphorylated NF (phNF) and found that, although total phNF immunofluorescence was consistent between motor neurons from the AB and the HB (Supplementary Figure S1), there was a significant increase in the number of phNF positive puncta along the neurites and in the cell bodies of the AB iPSC-derived motor neurons compared to the HB motor neurons (Figure 2E,F,H, arrows). This finding was supported by a subtle increase in insoluble total phNF, a 1.45 fold increase in insoluble phNF200, and 0.23 fold increase in phNF68 (Supplementary Figure S2A,B) in the AB motor neurons compared to HB, as analyzed by Western blot. There was no difference for phNF145 (Supplementary Figure S2A,B). Interestingly, there was an associated -0.51 fold decrease in the soluble phNF200 in the AB compared to the HB (Supplementary Figure S2A,C), further supporting a dramatic shift in soluble to insoluble protein. These data indicate that NF accumulation may be a contributing factor to motor neuron demise in the AB.

Considering that glial cells have been shown to contribute to ALS motor neuron toxicity and NF pathology [23-30], (Figure 1E), we next tested whether the presence of astrocyte and/or microglia-conditioned medium (ACM and MCM, respectively) could impact NF expression or accumulation. We differentiated HB and AB iPSCs into astrocytes and microglia (Supplementary Figure S3) and analyzed the ACM and MCM with a multiplex cytokine array (Figure 3). We then focused our analysis on the secreted factors above the level of detection. To our surprise, we found no difference between the secretome profile of HB ACM compared to AB ACM for anti-inflammatory cytokines or pro-inflammatory cytokines (Figure 3A,B). In contrast, we found that HB MCM produced significantly higher levels of the anti-inflammatory factors IL-1RA and IL-10 compared to the AB MCM (Figure 3C), but there was no difference between HB MCM and AB MCM in the key inflammatory molecule MCP-1 (Figure 3D). Although FGF2 was significantly upregulated in the AB MCM (Figure 3D), its overall expression level was low compared to MCP-1.

We next treated differentiated motor neurons with either HB ACM or AB ACM for $48 \mathrm{~h}$. Western blot analysis showed that neither HB ACM nor AB ACM altered NF protein levels for either $\mathrm{HB}$ or $\mathrm{AB}$ motor neurons (Figure $4 \mathrm{~A}-\mathrm{G}$ ), although there was a trend toward increased NF200 expression in the $\mathrm{HB}+\mathrm{AB}$ ACM condition (Figure 4D) and a trend toward decreased NF145 expression in the AB + HB ACM condition (Figure 4F). Interestingly, we did observe that both $\mathrm{HB} A C \mathrm{~A}$ and $\mathrm{AB} A C \mathrm{~A}$ induced a significant increase in phNF puncta in the $\mathrm{HB}$ motor neurons (Figure $4 \mathrm{H}, \mathrm{I}$ ), whereas neither HB ACM nor AB ACM induced a change in phNF puncta in the $A B$ motor neurons (Figure $4 \mathrm{~K}, \mathrm{~L}$ ), again despite no difference in total NF200 expression for $\mathrm{HB}$ and AB motor neurons treated with either HB ACM or AB ACM (Figure 4J,M). 

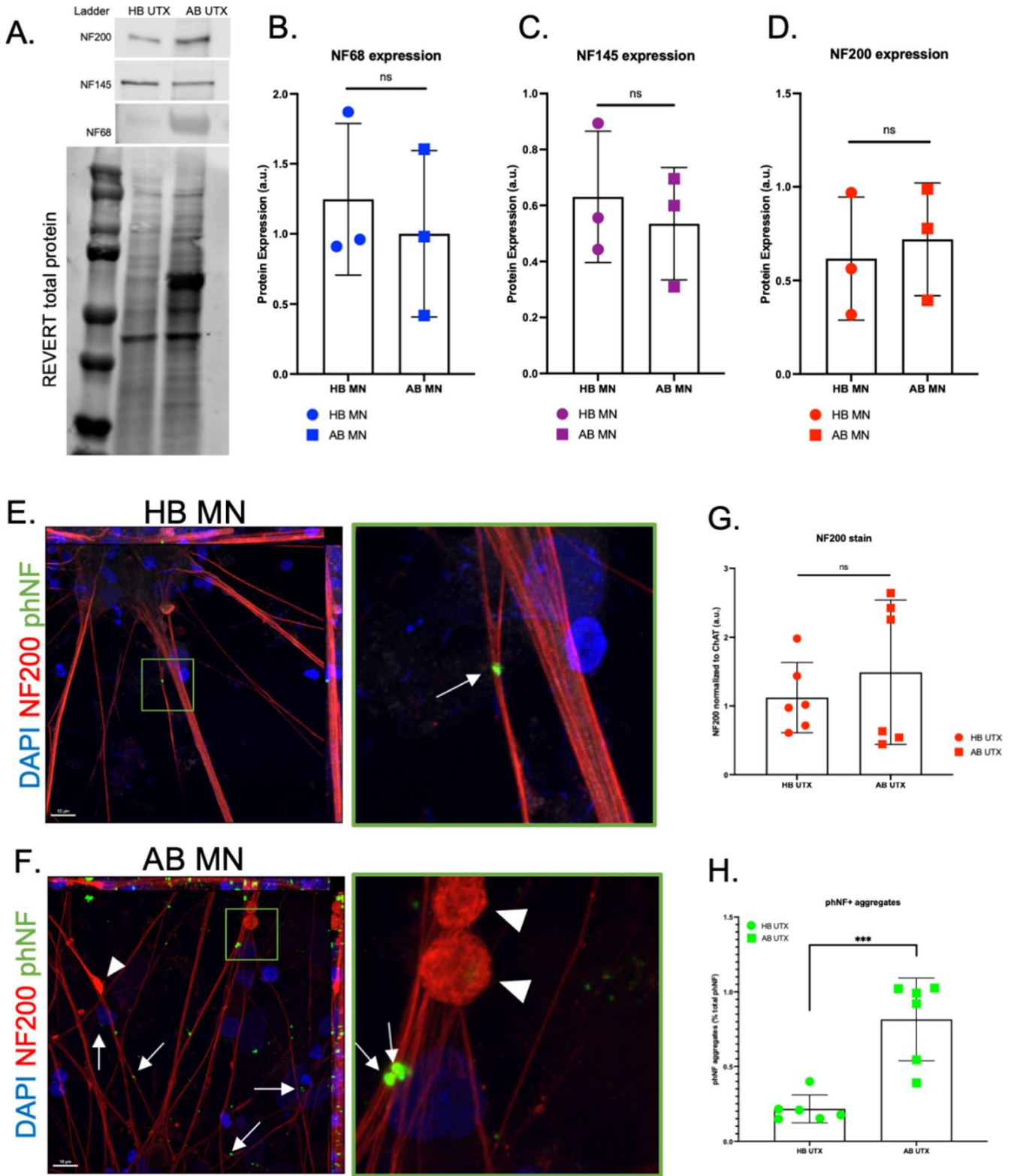

$\mathrm{H}$.

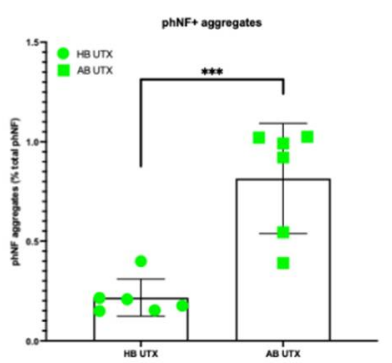

Figure 2. Aggregation of phosphorylated neurofilaments identified in $\mathrm{AB}$ motor neurons. (A-D) Western blot (WB) for NF68 (NF-L) (B), NF145 (NF-M) (C), and NF200 (NF-H) (D) subunits reveals no significant differences between $\mathrm{HB}$ and $\mathrm{AB} M \mathrm{MN}$ protein expression. (E,F) Representative immunocytochemistry (ICC) images of HB (E) and AB (F) motor neurons (MNs) (red = NF200, green $=$ phosphorylated NF $($ phNF), blue $=$ DAPI; $63 \times$ objective, scale bar $=10 \mu \mathrm{m})$. Green box is zoomed in panel on right. Arrows indicate aggregated phNF in neurites and cell bodies; arrowheads indicate neurite swellings. (G) Despite no significant differences between HB and AB for total NF200 expression, (H) quantification reveals significantly increased number of phNF+ aggregates in $\mathrm{AB}$ MNs compared to HB MNs [t-test, $\left.{ }^{* * *} p<0.0005\right]$. 
A.

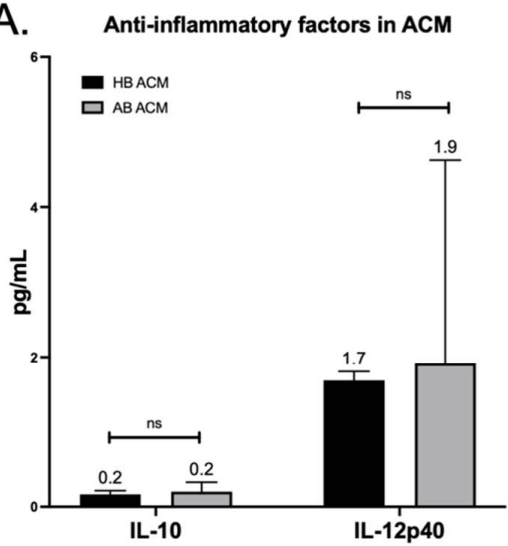

C. Anti-inflammatory factors in MCM

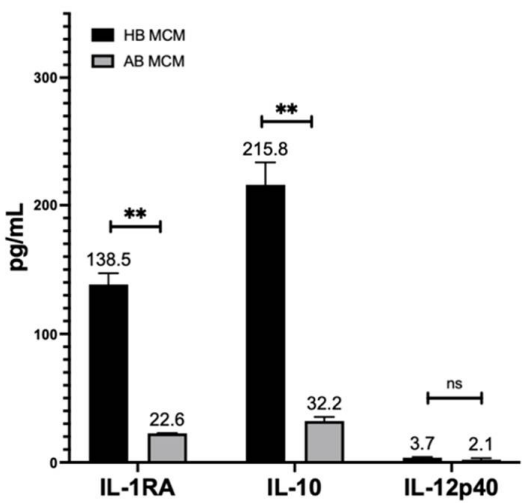

B. Pro-inflammatory factors in ACM

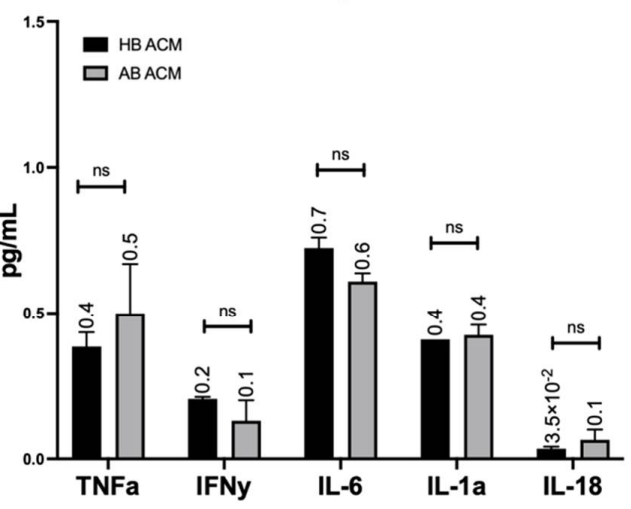

D.

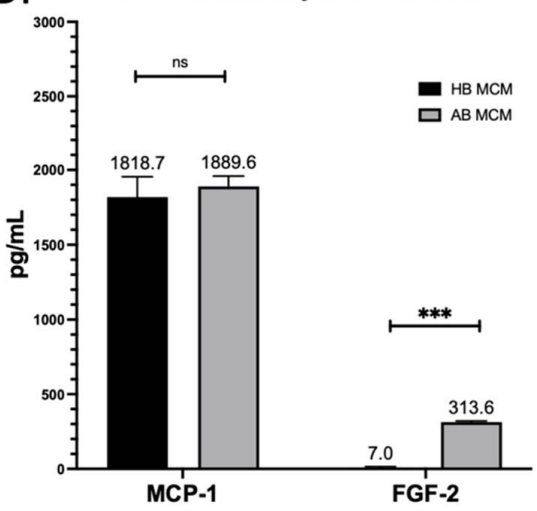

Figure 3. Differing secretome profiles identified between $\mathrm{HB}$ and $\mathrm{AB}$ microglia. (A) Multiplex cytokine array on HB (black bar) and AB (gray bar) ACM reveals similar levels of secreted antiinflammatory cytokines IL-10 and IL12p40 [t-tests, $\mathrm{ns}=0.7517,0.9155]$. (B) Similar levels of astrocyterelevant pro-inflammatory cytokines TNFa, IFNy, IL-6, IL-1a, and IL-18 were also noted between HB and AB ACM [ $t$-tests, $\mathrm{ns}=0.4547,0.2741,0.0695,9.6094,0.3604]$. (C) Multiplex cytokine array on HB (black bar) and AB (gray bar) MCM reveals significantly higher secreted levels of anti-inflammatory cytokines IL-1RA and IL-10, and no difference in IL12p40 by HB microglia compared to AB microglia [t-tests, first ${ }^{* *} p=0.0028$, second $\left.{ }^{* *} p=0.0046, \mathrm{~ns}=0.2348\right]$. (D) No significant difference in proinflammatory cytokine MCP-1, but FGF-2 was found to be secreted at higher levels in AB MCM than HB MCM [t-tests, $\left.\mathrm{ns}=0.5795,{ }^{* * *} p=0.0006\right]$.

We next tested how the addition of MCM would further impact NF expression and accumulation. IL-10 and IL-1 receptor antagonists (IL-1RA) prevent glial hyperactivation and IL-1 activation, respectively, thereby reducing overall inflammatory signaling [43,44]. Therefore, because we saw a significant increase in IL-10 and IL-1RA production in the HB MCM and no difference between HB ACM and AB ACM (Figures 3 and 4), we tested whether the addition of HB MCM to AB ACM would decrease motor neuron expression of phNF aggregates. We pre-treated AB astrocytes with HB MCM for $48 \mathrm{~h}$. We then changed the medium, collected AB ACM $48 \mathrm{~h}$ later, and added the HB MCM-treated AB ACM to either $\mathrm{HB}$ or $\mathrm{AB}$ motor neurons. HB MCM-AB ACM treatment had no overall effect on NF200 expression in the HB motor neurons (Figure 5A,B). Interestingly, this treatment did not show an increase in phNF puncta in the HB motor neurons (Figure 5A,C), which is in contrast to what was observed with $\mathrm{AB} A \mathrm{ACM}$ treatment alone (Figure $4 \mathrm{I}$ ). HB MCM-AB ACM treatment on the AB motor neurons also did not induce a change in overall NF200 expression (Figure 5D,E), but this treatment did significantly reduce the presence of phNF puncta in the $\mathrm{AB}$ motor neuron cultures (Figure 5D,F). Together, these data suggest that factors released from the HB microglia are involved in modulating phNF deposition in ALS motor neurons. 
A.

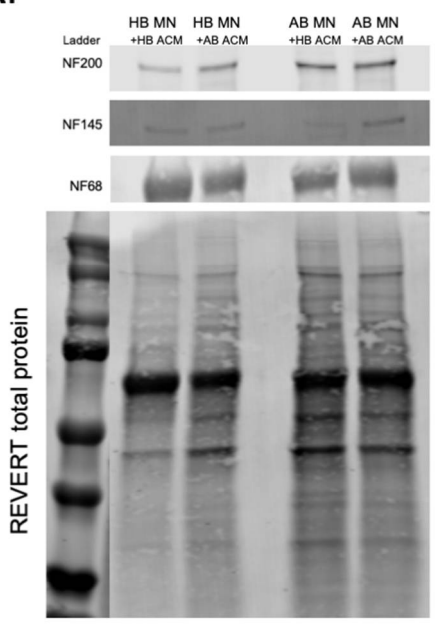

B.
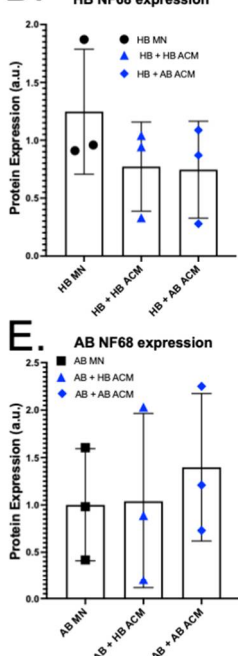

H. $H B M N+H B A C M$

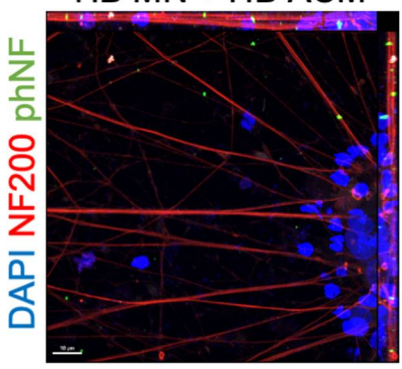

$\mathrm{HB} M \mathrm{MN}+\mathrm{AB} A \mathrm{ACM}$

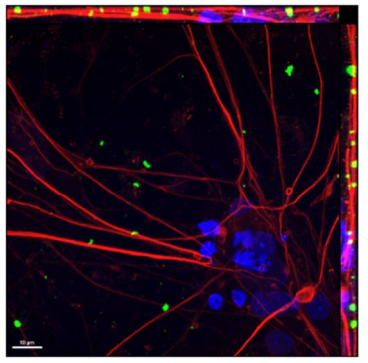

K. $A B M N+H B A C M$

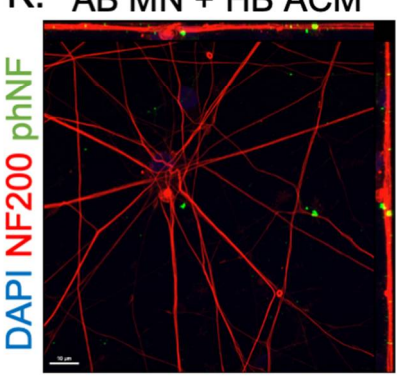

$A B M N+A B A C M$

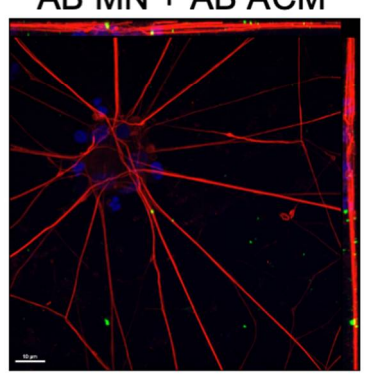

C.

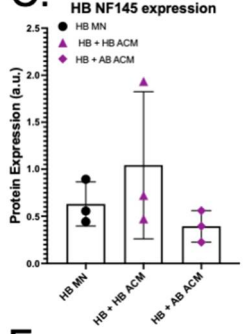

$\mathrm{F}$.
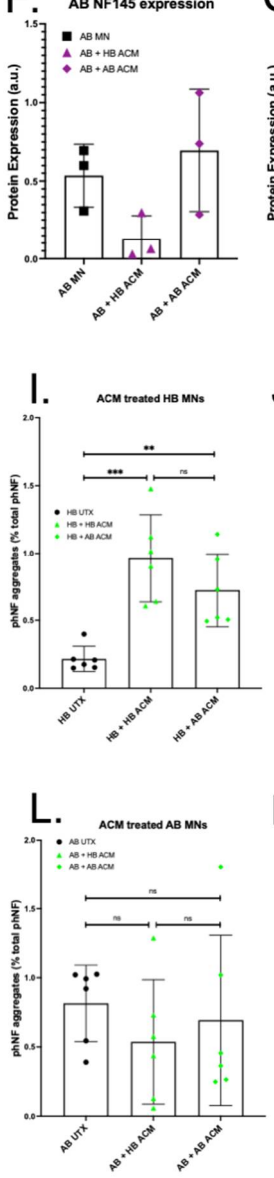

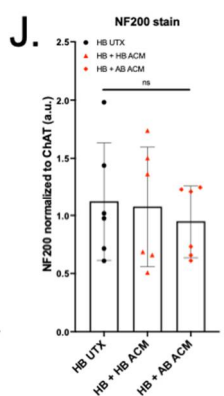

D.

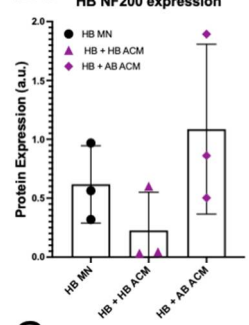

G.

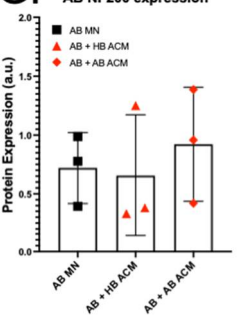

M.

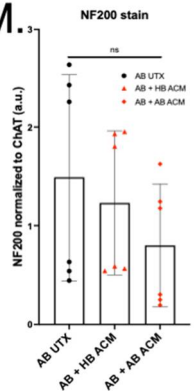

Figure 4. Effect of $\mathrm{HB}$ and $\mathrm{AB}$ astrocyte-conditioned media on NF subunit expression and phNF+ aggregation. (A-G) WB for NF68, NF145, and NF200 subunits reveal no significant changes after treatment with $\mathrm{HB}$ or $\mathrm{AB}$ astrocyte-conditioned media (ACM) on HB MNs (B-D) or AB MNs (E-G). Untreated (UTX, black) samples are copied from Figure 2 for ease of comparison to ACM treatment. WB of UTX samples run simultaneously with ACM-treated samples to allow for direct comparison. Full gel showing all lanes is available in Supplemental Figure S4. (H) Representative images of HB MN ICC after HB (left) or AB (right) ACM treatment (red $=$ NF200, green $=p h N F$, blue = DAPI, $63 \times$ objective, scale bars $=10 \mu \mathrm{m}$ ). ICC, imaging, and analyses performed simultaneously with UTX samples shown in Figure 2 was carried out for direct comparison. (I) Quantification for phNF in $\mathrm{HB}$ MNs shows a significant increase in phNF+ aggregates after ACM treatment from either $\mathrm{HB}$ or $\mathrm{AB}$ ACM with no significant difference between ACM treatments [1-way ANOVA, ${ }^{* * *} p=0.0003$, $* * p=0.0083, \mathrm{~ns}=0.2579$ ]. (J) ICC for NF200 in HB confirms no significant changes after HB or $\mathrm{AB} A C M$ treatment. (K) Representative images of $\mathrm{AB}$ MNs after $\mathrm{HB}$ (left) or $\mathrm{AB}$ (right) $\mathrm{ACM}$ $($ red $=$ NF200, green $=$ phNF, blue $=$ DAPI, $63 \times$ objective, scale bars $=10 \mu \mathrm{m}) .($ L) Quantification showed no change in phNF+ aggregation in $\mathrm{AB}$ MNs after treatment with either $\mathrm{HB}$ or $\mathrm{AB} \mathrm{ACM}$ [1-way ANOVA, ns $=0.5683,0.8932,0.8335]$. (M) ICC for NF200 in AB MNs confirms no significant changes after $\mathrm{HB} \mathrm{ACM}$ or $\mathrm{AB} A \mathrm{ACM}$ treatment. 
A.

$\mathrm{HB} M \mathrm{MN}+(\mathrm{HB} M C M->\mathrm{AB} A C M)$
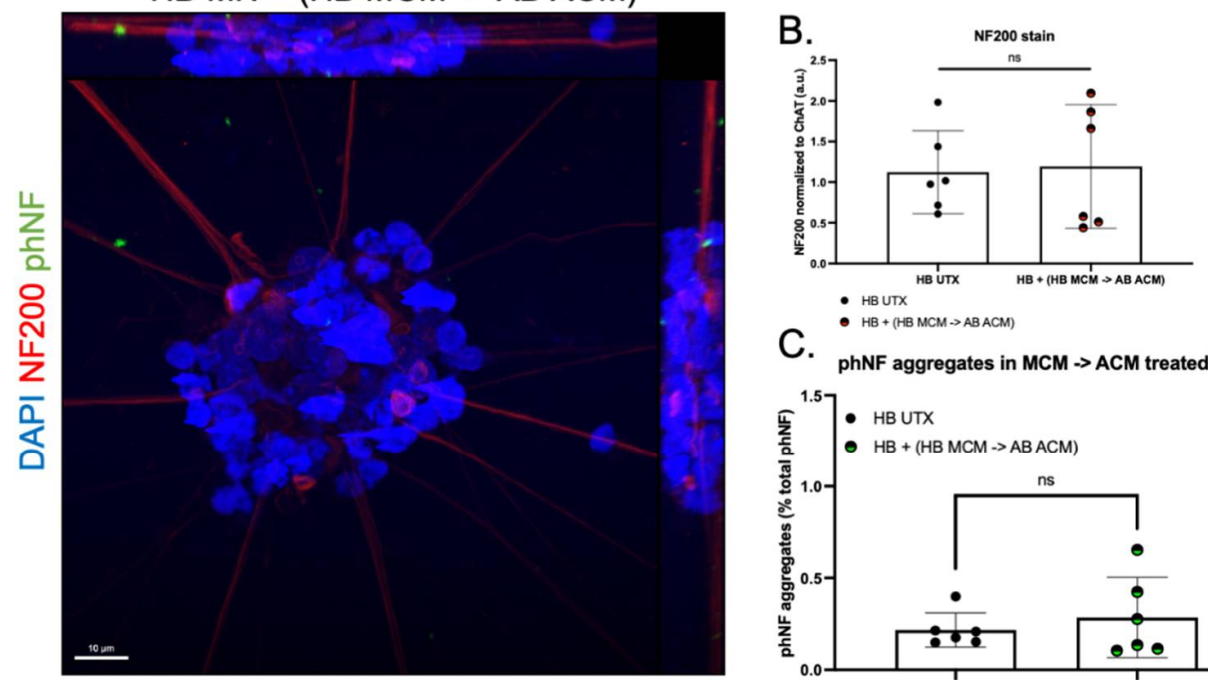

C.

phNF aggregates in MCM $\rightarrow$ ACM treated MNs

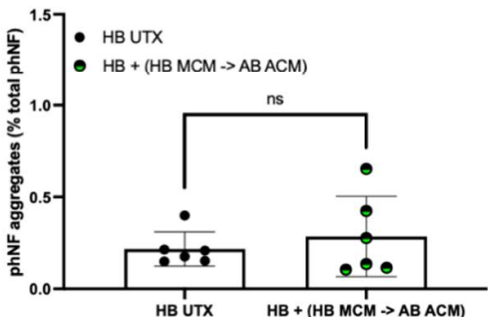

D.

$A B M N+(H B M C M-A B A C M)$
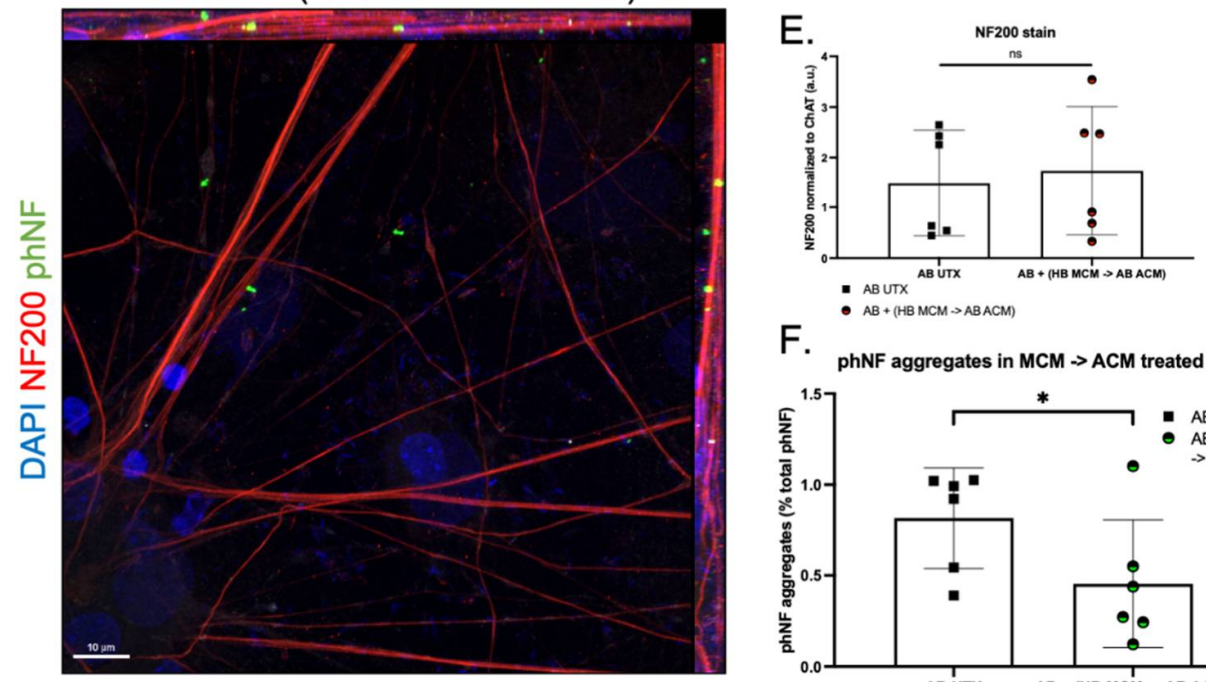

$\mathrm{F}$

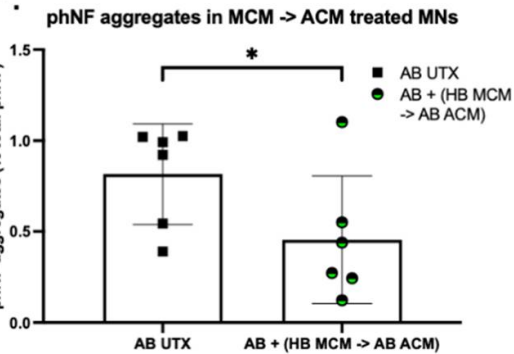

Figure 5. Effect of $\mathrm{HB}$ and $\mathrm{AB}$ microglia-conditioned media on ACM-induced ALS phenotype. (A) Representative image of $\mathrm{HB}$ motor neurons (MNs) treated with medium from $\mathrm{AB}$ astrocytes pretreated with HB microglia-conditioned media $(\mathrm{HB}$ MCM) (red = NF200, green = phNF, blue = DAPI $63 \times$ objective, scale bars $=10 \mu \mathrm{m}$ ). (B) Quantification confirms no change in NF subunit expression in $\mathrm{HB}$ MNs following treatment with $\mathrm{AB}$ ACM pre-treated with HB MCM [ $t$-test, ns $=0.7795]$. (C) Quantification shows prevention of ACM induced phNF+ aggregates in HB MNs after pretreatment of $\mathrm{AB}$ astrocytes with $\mathrm{HB}$ MCM [t-test, ns $=0.4340]$. (D) Representative image of $\mathrm{AB}$ MNs treated with AB ACM pre-treated with HB MCM (red = NF200, green = phNF, blue = DAPI $63 \times$ objective). (E) Quantification confirms no change in NF subunit expression in AB MNs after application of AB ACM pre-treated with HB MCM [t-test, ns $=0.3222]$. (F) Quantification reveals a significant reduction in phNF+ aggregates when $\mathrm{HB} M C M$ pre-treated $\mathrm{AB} A C M$ is applied to $\mathrm{AB}$ MNs $\left[t\right.$-test, $\left.{ }^{*}=0.0203\right]$.

\section{Discussion}

ALS is an always-fatal disease that currently lacks effective treatment options. Vast amounts of work have focused on elucidating the molecular mechanisms associated with motor neuron loss in order to identify novel therapeutic targets. Among these studies, data suggest that both phNF aggregation and glial-neuron interactions can contribute to motor neuron malfunction and loss in ALS [1,23]. In the current study, we found that 
glial-produced factors can augment phNF accumulation suggesting a link between these two pathogenic pathways.

Axonal swellings were identified in postmortem tissue from a motor neuron disease patient in the late 1960s [45] with subsequent studies identifying neurofilament accumulation as a pathological property of ALS [5]. However, there are contrasting data across in vitro, in vivo, and human studies regarding whether NF levels are upregulated or downregulated in ALS and the relationship to disease. For example, neuronal NF overexpression in mice induces an ALS-like phenotype [46,47], NF deletion in SOD1 mice is protective [48], and NF levels are significantly increased in the CSF and blood of ALS patients [12]. In contrast, others have found that NF levels are decreased in mutant SOD1 iPSC-derived motor neurons [20] and crossing mutant SOD1 mice with mice overexpressing NF-L or NF-H reduces disease phenotypes and extends lifespan [49,50]. Although we found significantly elevated transcript levels of NF-L, NF-M, and NF-H in the AB motor neurons (Figure 1), this did not translate into elevated protein levels (Figure 2). This discordance could be due to cellular regulation of protein levels through microRNAs and/or protein quality control mechanisms $[4,51,52]$. Nevertheless, we did still observe an increased abundance of phosphorylated NF accumulations in the AB motor neurons (Figure 2). As such, it may be less about the overall protein abundance and more about altered subunit dynamics leading to aggregation, disrupted downstream signaling, and, therefore, hyperphosphorylation $[11,20]$. As NFs are essential for structural stability, nerve conduction velocity, organelle interactions, and synaptic function [53], even subtle disruptions could have profound consequences for neuronal health and survival.

Astrocytes and microglia are integral components of a normally functioning nervous system. Astrocytes, a highly abundant cell type in the central nervous system, perform a wide range of functions, including providing trophic support to surrounding cells, maintaining extracellular ion balance, secreting molecules necessary for neuronal energy and metabolism, and taking up and recycling neurotransmitters [54]. Microglia, the resident immune cells of the central nervous system, continuously survey the environment to maintain synaptic function and debris clearance [55]. However, injury or disease can cause both astrocytes and microglia to alter their function and upregulate inflammatory signaling. In this regard, studies have found that both mutant SOD1-expressing mice and mutant OPTN-expressing mice upregulate NFKB expression that drives production of IL-6, IL-1 $\beta$, and TNF $\alpha$ from both astrocytes and microglia [31,56], which overlaps with cytokines observed in the iPSC-derived ACM (Figure 3). Previous studies have clearly demonstrated that familial and sporadic ALS iPSC-derived astrocytes induce motor neuron loss [57], but much less is known about the specific astrocyte secreted proteins involved, the toxic nature of the ALS microglial secretome, or how combined astrocyte-microglia signaling can influence motor neuron health and survival in ALS.

What we found surprising, though, was that the astrocyte cytokine profile for the $\mathrm{HB}$ was very similar to the AB (Figure 3). Given the robust toxic effects of ALS astrocytes in other models, we hypothesized that we would find an increase in pro-inflammatory cytokines and/or a decrease in the anti-inflammatory cytokines in the AB ACM compared to the HB ACM. However, since we did not see a difference between the ACM cytokine profiles, it was then not surprising that both the HB ACM and the AB ACM induced an upregulation of phNF in the HB motor neurons (Figure 4). These data suggest that the HB motor neurons are capable of developing an ALS-like phenotype, but perhaps other systemic signaling processes are keeping them from tipping to a more pathological state. This idea is supported by our data showing that pre-treating astrocytes with $\mathrm{HB}$ MCM reduced the phNF deposition (Figure 5). Although the cytokine data presented here (Figure 3) focused on a small number of factors that were above the level of detection in the assay, it is likely that there are other factors involved in the microglia-to-astrocyte signaling. In this regard, additional analysis of the motor neuron RNAseq data (Figure 1), as well as generating RNAseq data from ACM-MCM-treated motor neurons, will be informative for future pathway analysis studies. 
Finally, our study focused on a unique discordant identical twin pair to model sporadic ALS using the HB as an approximation of an isogenic cell line. Therefore, we are not able to generalize these data to other sporadic ALS cases or to familial ALS cases. Future studies will be needed to better characterize the glial-motor neuron interplay in ALS more broadly, but our data provide an interesting starting point for assessing how microglial-astrocytemotor neuron interactions contribute to ALS pathology.

\section{Conclusions}

Using a unique set of iPSCs to model sporadic ALS, we found that ALS-affected motor neurons exhibit increased expression of insoluble NF and the presence of NF accumulations in both the cell body and along neurites. Moreover, our study also found that secreted factors from microglia and astrocytes impact motor neuron NF accumulation. Together, these data further implicate astrocytes and microglia in ALS pathology and suggest a potential role for pro-inflammatory and anti-inflammatory cytokine signaling in motor neuron disease.

Supplementary Materials: The following supporting information can be downloaded at: https: / / www.mdpi.com/article/10.3390/genes13020241/s1, Figure S1: Only phNF+ aggregates differ between treatment groups - not total phNF-indicative of an ALS phenotype, Figure S2: Soluble and insoluble fractionation of $\mathrm{HB}$ and $\mathrm{AB}$ MN pellets, Figure S3: Representative images of astrocytes and microglia differentiated from iPSCs, Figure S4: Full Western blots showing untreated and treated MN samples to allow for direct comparison.

Author Contributions: Conceptualization, R.L.A. and A.D.E.; Methodology, R.L.A., J.W.A., J.A., R.A.U., M.T.Z. and A.J.M.; Data Analysis, R.L.A., J.W.A., J.A., M.T.Z. and A.J.M.; Resources, R.A.U. and A.D.E.; Writing-Original Draft Preparation, R.L.A., J.W.A. and A.D.E.; Writing-Review \& Editing, R.L.A., J.W.A., J.A., R.A.U., M.T.Z., A.J.M. and A.D.E.; Funding Acquisition, A.D.E. All authors have read and agreed to the published version of the manuscript.

Funding: This work was funded in part by the Phoebe Lewis Regenerative Medicine Fund and grants from the Neuroscience Research Center and the Center for Immunology at the Medical College of Wisconsin.

Institutional Review Board Statement: iPSC lines were previously generated from identical twins discordant for ALS under an approved IRB protocol (PRO00024167).

Data Availability Statement: Complete RNAseq data have been deposited in the Gene Expression Omnibus (GEO) database (GEO accession number GSE192755).

Acknowledgments: The authors thank the Froedtert Hospital ALS Clinic for initially providing the patient samples and Mike Dwinell for helpful discussions.

Conflicts of Interest: The authors declare no conflict of interest.

\section{References}

1. Dhasmana, S.; Dhasmana, A.; Narula, A.S.; Jaggi, M.; Yallapu, M.M.; Chauhan, S.C. The panoramic view of amyotrophic lateral sclerosis: A fatal intricate neurological disorder. Life Sci. 2022, 288, 120156. [CrossRef] [PubMed]

2. Mizuno, Y.; Amari, M.; Takatama, M.; Aizawa, H.; Mihara, B.; Okamoto, K. Immunoreactivities of p62, an ubiqutin-binding protein, in the spinal anterior horn cells of patients with amyotrophic lateral sclerosis. J. Neurol. Sci. 2006, 249, 13-18. [CrossRef] [PubMed]

3. Neumann, M.; Sampathu, D.M.; Kwong, L.K.; Truax, A.C.; Micsenyi, M.C.; Chou, T.T.; Bruce, J.; Schuck, T.; Grossman, M.; Clark, C.M.; et al. Ubiquitinated TDP-43 in Frontotemporal Lobar Degeneration and Amyotrophic Lateral Sclerosis. Science 2006, 314, 130-133. [CrossRef] [PubMed]

4. Seminary, E.R.; Sison, S.L.; Ebert, A.D. Modeling Protein Aggregation and the Heat Shock Response in ALS iPSC-Derived Motor Neurons. Front. Neurosci. 2018, 12, 86. [CrossRef] [PubMed]

5. Figlewicz, D.A.; Krizus, A.; Martinoli, M.-G.; Meininger, V.; Dib, M.; Rouleau, G.A.; Julien, J.-P. Variants of the heavy neurofilament subunit are associated with the development of amyotrophic lateral sclerosis. Hum. Mol. Genet. 1994, 3, 1757-1761. [CrossRef]

6. Cummings, C.J.; Mancini, M.A.; Antalffy, B.; DeFranco, D.B.; Orr, H.; Zoghbi, H. Chaperone suppression of aggregation and altered subcellular proteasome localization imply protein misfolding in SCA1. Nat. Genet. 1998, 19, 148-154. [CrossRef] [PubMed] 
7. Ii, K.; Ito, H.; Tanaka, K.; Hirano, A. Immunocytochemical co-localization of the proteasome in ubiquitinated structures in neu-rodegenerative diseases and the elderly. J. Neuropathol. Exp. Neurol. 1997, 56, 125-131. [CrossRef]

8. Behl, C.; Davis, J.B.; Lesley, R.; Schubert, D. Hydrogen peroxide mediates amyloid beta protein toxicity. Cell 1994, 77, 817-827. [CrossRef]

9. Hsu, L.J.; Sagara, Y.; Arroyo, A.; Rockenstein, E.; Sisk, A.; Mallory, M.; Wong, J.; Takenouchi, T.; Hashimoto, M.; Masliah, E. $\alpha$-Synuclein Promotes Mitochondrial Deficit and Oxidative Stress. Am. J. Pathol. 2000, 157, 401-410. [CrossRef]

10. Rosengren, L.E.; Karlsson, J.E.; Karlsson, J.O.; Persson, L.I.; Wikkelso, C. Patients with amyotrophic lateral sclerosis and other neu-rodegenerative diseases have increased levels of neurofilament protein in CSF. J. Neurochem. 1996, 67, 2013-2018. [CrossRef]

11. Zucchi, E.; Bonetto, V.; Sorarù, G.; Martinelli, I.; Parchi, P.; Liguori, R.; Mandrioli, J. Neurofilaments in motor neuron disorders: Towards promising diagnostic and prognostic biomarkers. Mol. Neurodegener. 2020, 15, 58. [CrossRef] [PubMed]

12. Sferruzza, G.; Bosco, L.; Falzone, Y.M.; Russo, T.; Domi, T.; Quattrini, A.; Filippi, M.; Riva, N. Neurofilament light chain as a biological marker for amyotrophic lateral sclerosis: A meta-analysis study. Amyotroph. Lateral Scler. Front. Degener. 2021, 1-12. [CrossRef] [PubMed]

13. Anderton, B.H.; Ayers, M.; Thorpe, R. Neurofilaments from mammalian central and peripheral nerve share certain polypeptides. FEBS Lett. 1978, 96, 159-163. [CrossRef]

14. Julien, J.P.; Mushynski, W.E. The distribution of phosphorylation sites among identified proteolytic fragments of mammalian neurofilaments. J. Biol. Chem. 1983, 258, 4019-4025. [CrossRef]

15. Bocquet, A.; Berges, R.; Frank, R.; Robert, P.; Peterson, A.C.; Eyer, J. Neurofilaments Bind Tubulin and Modulate Its Polymerization. J. Neurosci. 2009, 29, 11043-11054. [CrossRef]

16. Schwartz, M.L.; Shneidman, P.S.; Bruce, J.; Schlaepfer, W.W. Stabilization of neurofilament transcripts during postnatal development. Brain Res. Mol. Brain Res. 1994, 27, 215-220. [CrossRef]

17. Beaulieu, J.-M.; Nguyen, M.D.; Julien, J.-P. Late Onset Death of Motor Neurons in Mice Overexpressing Wild-Type Peripherin. J. Cell Biol. 1999, 147, 531-544. [CrossRef]

18. Križ, J.; Zhu, Q.; Julien, J.-P.; Padjen, A.L. Electrophysiological properties of axons in mice lacking neurofilament subunit genes: Disparity between conduction velocity and axon diameter in absence of NF-H. Brain Res. 2000, 885, 32-44. [CrossRef]

19. Sun, X.; Song, J.; Huang, H.; Chen, H.; Qian, K. Modeling hallmark pathology using motor neurons derived from the family and sporadic amyotrophic lateral sclerosis patient-specific iPS cells. Stem Cell Res. Ther. 2018, 9, 315. [CrossRef]

20. Chen, H.; Qian, K.; Du, Z.; Cao, J.; Petersen, A.; Liu, H.; Blackbourn, L.W.; Huang, C.-L.; Errigo, A.; Yin, Y.; et al. Modeling ALS with iPSCs Reveals that Mutant SOD1 Misregulates Neurofilament Balance in Motor Neurons. Cell Stem Cell 2014, 14, 796-809. [CrossRef]

21. Qian, K.; Huang, H.; Peterson, A.; Hu, B.; Maragakis, N.J.; Ming, G.-L.; Chen, H.; Zhang, S.-C. Sporadic ALS Astrocytes Induce Neuronal Degeneration In Vivo. Stem Cell Rep. 2017, 8, 843-855. [CrossRef] [PubMed]

22. Ackerley, S.; Grierson, A.; Brownlees, J.; Thornhill, P.; Anderton, B.H.; Leigh, P.N.; Shaw, C.E.; Miller, C. Glutamate Slows Axonal Transport of Neurofilaments in Transfected Neurons. J. Cell Biol. 2000, 150, 165-176. [CrossRef]

23. Julien, J.-P. Amyotrophic Lateral Sclerosis: Unfolding the Toxicity of the Misfolded. Cell 2001, 104, 581-591. [CrossRef]

24. Lasiene, J.; Yamanaka, K. Glial Cells in Amyotrophic Lateral Sclerosis. Neurol. Res. Int. 2011, 2011, 718987. [CrossRef]

25. Zhao, C.; Devlin, A.C.; Chouhan, A.K.; Selvaraj, B.T.; Stavrou, M.; Burr, K.; Brivio, V.; He, X.; Mehta, A.R.; Story, D.; et al. Mutant C9orf72 human iPSC-derived astrocytes cause non-cell autonomous motor neuron pathophysiology. Glia 2020, 68, 1046-1064. [CrossRef] [PubMed]

26. Birger, A.; Ben-Dor, I.; Ottolenghi, M.; Turetsky, T.; Gil, Y.; Sweetat, S.; Perez, L.; Belzer, V.; Casden, N.; Steiner, D.; et al. Human iPSC-derived astrocytes from ALS patients with mutated C9ORF72 show increased oxidative stress and neurotoxicity. EBioMedicine 2019, 50, 274-289. [CrossRef] [PubMed]

27. Clement, A.M.; Nguyen, M.D.; Roberts, E.A.; Garcia, M.L.; Boillee, S.; Rule, M.; McMahon, A.P.; Doucette, W.; Siwek, D.; Ferrante, R.J.; et al. Wild-Type Nonneuronal Cells Extend Survival of SOD1 Mutant Motor Neurons in ALS Mice. Science 2003, 302, 113-117. [CrossRef] [PubMed]

28. Di Giorgio, F.P.; Carrasco, M.A.; Siao, M.C.; Maniatis, T.; Eggan, K. Non-cell autonomous effect of glia on motor neurons in an embryonic stem cell-based ALS model. Nat. Neurosci. 2007, 10, 608-614. [CrossRef]

29. Marchetto, M.C.; Muotri, A.R.; Mu, Y.; Smith, A.M.; Cezar, G.G.; Gage, F.H. Non-Cell-Autonomous Effect of Human SOD1G37R Astrocytes on Motor Neurons Derived from Human Embryonic Stem Cells. Cell Stem Cell 2008, 3, 649-657. [CrossRef]

30. Benkler, C.; Ben-Zur, T.; Barhum, Y.; Offen, D. Altered astrocytic response to activation in SOD1(G93A) mice and its implications on amyotrophic lateral sclerosis pathogenesis. Glia 2013, 61, 312-326. [CrossRef]

31. Frakes, A.E.; Ferraiuolo, L.; Haidet-Phillips, A.M.; Schmelzer, L.; Braun, L.; Miranda, C.J.; Ladner, K.J.; Bevan, A.K.; Foust, K.D.; Godbout, J.P.; et al. Microglia induce motor neuron death via the classical NF-кB pathway in amyotrophic lateral sclerosis. Neuron 2014, 81, 1009-1023. [CrossRef] [PubMed]

32. Spiller, K.J.; Restrepo, C.; Khan, T.; Dominique, M.A.; Fang, T.C.; Canter, R.G.; Roberts, C.J.; Miller, K.R.; Ransohoff, R.M.; Trojanowski, J.Q.; et al. Microglia-Mediated recovery from ALS-relevant motor neuron degeneration in a mouse model of TDP-43 proteinopathy. Nat. Neurosci. 2018, 21, 329-340. [CrossRef] [PubMed] 
33. Deora, V.; Lee, J.D.; Albornoz, E.A.; McAlary, L.; Jagaraj, C.J.; Robertson, A.A.B.; Atkin, J.; Cooper, M.A.; Schroder, K.; Yerbury, J.; et al. The microglial NLRP3 inflammasome is activated by amyotrophic lateral sclerosis proteins. Glia 2020, 68, 407-421. [CrossRef] [PubMed]

34. Clarke, B.E.; Patani, R. The microglial component of amyotrophic lateral sclerosis. Brain 2020, 143, 3526-3539. [CrossRef] [PubMed]

35. Liddelow, S.A.; Guttenplan, K.A.; Clarke, L.E.; Bennett, F.C.; Bohlen, C.J.; Schirmer, L.; Bennett, M.L.; Münch, A.E.; Chung, W.-S.; Peterson, T.C.; et al. Neurotoxic reactive astrocytes are induced by activated microglia. Nature 2017, 541, 481-487. [CrossRef] [PubMed]

36. Seminary, E.R.; Santarriaga, S.; Wheeler, L.; Mejaki, M.; Abrudan, J.; Demos, W.; Zimmermann, M.T.; Urrutia, R.A.; Fee, D.; Barkhaus, P.E.; et al. Motor Neuron Generation from iPSCs from Identical Twins Discordant for Amyotrophic Lateral Sclerosis. Cells 2020, 9, 571. [CrossRef]

37. Maury, Y.; Côme, J.; Piskorowski, R.A.; Salah-Mohellibi, N.; Chevaleyre, V.; Peschanski, M.; Martinat, C.; Nedelec, S. Combinatorial analysis of developmental cues efficiently converts human plu-ripotent stem cells into multiple neuronal subtypes. Nat. Biotechnol. 2015, 33, 89-96. [CrossRef]

38. Allison, R.; Welby, E.; Khayrullina, G.; Burnett, B.; Ebert, A. Viral mediated knockdown of GATA6 in SMA iPSC-derived astrocytes prevents motor neuron loss and microglial activation. bioRxiv 2021. [CrossRef]

39. McQuade, A.; Coburn, M.; Tu, C.H.; Hasselmann, J.; Davtyan, H.; Blurton-Jones, M. Development and validation of a simplified method to generate human microglia from pluripotent stem cells. Mol. Neurodegener. 2018, 13, 67. [CrossRef]

40. Kalari, K.R.; Nair, A.A.; Bhavsar, J.D.; O’Brien, D.R.; Davila, J.I.; Bockol, M.A.; Nie, J.; Tang, X.; Baheti, S.; Doughty, J.B.; et al MAP-RSeq: Mayo Analysis Pipeline for RNA sequencing. BMC Bioinform. 2014, 15, 224. [CrossRef]

41. Robinson, M.D.; McCarthy, D.J.; Smyth, G.K. EdgeR: A Bioconductor package for differential expression analysis of digital gene expression data. Bioinformatics 2010, 26, 139-140. [CrossRef] [PubMed]

42. Santarriaga, S.; Luecke, I.; Ebert, A. Detection of soluble and insoluble protein species in patient-derived iPSCs. In Methods in Molecular Biology; Springer-Nature: Berlin/Heidelberg, Germany, 2022; in press.

43. Liao, Z.; Grimshaw, R.S.; Rosenstreich, D.L. Identification of a specific interleukin 1 inhibitor in the urine of febrile patients. J. Exp. Med. 1984, 159, 126-136. [CrossRef] [PubMed]

44. Shemer, A.; Scheyltjens, I.; Frumer, G.R.; Kim, J.S.; Grozovski, J.; Ayanaw, S.; Dassa, B.; Van Hove, H.; Chappell-Maor, L.; Boura-Halfon, S.; et al. Interleukin-10 Prevents Pathological Microglia Hyperactivation following Peripheral Endotoxin Challenge. Immunity 2020, 53, 1033-1049.e7. [CrossRef] [PubMed]

45. Carpenter, S. Proximal axonal enlargement in motor neuron disease. Neurology 1968, 18, 841. [CrossRef]

46. Côté, F.; Collard, J.F.; Julien, J.P. Progressive neuronopathy in transgenic mice expressing the human neurofilament heavy gene: A mouse model of amyotrophic lateral sclerosis. Cell 1993, 73, 35-46. [CrossRef]

47. Xu, Z.; Cork, L.C.; Griffin, J.W.; Cleveland, D.W. Increased expression of neurofilament subunit NF-L produces morphological alterations that resemble the pathology of human motor neuron disease. Cell 1993, 73, 23-33. [CrossRef]

48. Williamson, T.L.; Bruijn, L.I.; Zhu, Q.; Anderson, K.L.; Anderson, S.D.; Julien, J.-P.; Cleveland, D. Absence of neurofilaments reduce the selective vulnerability of motor neurons and slows disease caused by a familial amyotrophic lateral sclerosis-linked superoxide dismutase 1 mutant. Proc. Natl. Acad. Sci. USA 1998, 95, 9631-9636. [CrossRef]

49. Couillard-Despres, S.; Zhu, Q.; Wong, P.C.; Price, D.L.; Cleveland, D.; Julien, J.-P. Protective effect of neurofilament heavy gene overexpression in motor neuron disease induced by mutant superoxide dismutase. Proc. Natl. Acad. Sci. USA 1998, 95, 9626-9630. [CrossRef]

50. Kong, J.; Xu, Z. Overexpression of neurofilament subunit NF-L and NF-H extends survival of a mouse model for amyotrophic lateral sclerosis. Neurosci. Lett. 2000, 281, 72-74. [CrossRef]

51. Campos-Melo, D.; Hawley, Z.C.E.; Strong, M.J. Dysregulation of human NEFM and NEFH mRNA stability by ALS-linked miRNAs. Mol. Brain 2018, 11, 43. [CrossRef]

52. Shahheydari, H.; Ragagnin, A.; Walker, A.; Toth, R.P.; Vidal, M.; Jagaraj, C.J.; Perri, E.R.; Konopka, A.; Sultana, J.M.; Atkin, J.D. Protein Quality Control and the Amyotrophic Lateral Sclerosis/Frontotemporal Dementia Continuum. Front. Mol. Neurosci. 2017, 10, 119. [CrossRef] [PubMed]

53. Yuan, A.; Rao, M.; Veeranna; Nixon, R.A. Neurofilaments and Neurofilament Proteins in Health and Disease. Cold Spring Harb. Perspect. Biol. 2017, 9, a018309. [CrossRef] [PubMed]

54. Wang, D.D.; Bordey, A. The astrocyte odyssey. Prog. Neurobiol. 2008, 86, 342-367. [CrossRef] [PubMed]

55. Yang, Z.-Y.; Jin, W.-L.; Xu, Y.; Jin, M.-Z. Microglia in neurodegenerative diseases. Neural Regen. Res. 2021, 16, 270-280. [CrossRef]

56. Liu, Z.; Li, H.; Hong, C.; Chen, M.; Yue, T.; Chen, C.; Wang, Z.; You, Q.; Li, C.; Weng, Q.; et al. ALS-Associated E478G Mutation in Human OPTN (Optineurin) Promotes Inflammation and Induces Neuronal Cell Death. Front. Immunol. 2018, 9, 2647. [CrossRef]

57. Van Harten, A.C.M.; Phatnani, H.; Przedborski, S. Non-Cell-Autonomous pathogenic mechanisms in amyotrophic lateral sclerosis. Trends Neurosci. 2021, 44, 658-668. [CrossRef] 